\title{
Calcium/Calmodulin-Dependent Protein Kinase II Alters Structural Plasticity and Cytoskeletal Dynamics in Drosophila
}

\author{
Ryan Andersen, Yimei Li, Mary Resseguie, and Jay E. Brenman \\ Department of Cell and Developmental Biology and Neuroscience Center, University of North Carolina Chapel Hill, Chapel Hill, North Carolina 27599
}

\begin{abstract}
Drosophila dendritic arborization (da) neurons contain subclasses of neurons with distinct dendritic morphologies. We investigated calcium/calmodulin-dependent protein kinase II (CaMKII) regulation of dendritic structure and dynamics in vivo using optically transparent Drosophila larvae. CaMKII increases the dynamic nature and formation of dendritic filopodia throughout larval development but only affects neurons that normally contain dendritic filopodia. In parallel, we examined the effects of Racl activity on dendritic structure to explore signaling specificity. In contrast to CaMKII activity, Rac1 does not alter filopodia stability but instead causes de novo filopodia formation on all da neurons. Although both mediators increase cytoskeletal turnover, measured by fluorescence recovery after photobleaching experiments, only CaMKII increases the dynamic nature of dendritic filopodia. CaMKII signaling thus appears to use mechanisms and machinery distinct from Rac1 signaling. This study illustrates a molecular means of uncoupling cytoskeletal regulation from morphological regulation. Our results suggest that Drosophila dendritic filopodia may share some cytoskeletal regulatory mechanisms with mammalian dendritic filopodia. Furthermore, general dendrite cytoskeletal compartmentalization is conserved in multipolar neurons.
\end{abstract}

Key words: actin; dendrites; Drosophila; filopodia; Rac1; CaMKII

\section{Introduction}

Neuronal dendrites process and receive information, either from other neurons or the external environment. Regulation of dendritic morphological diversity is a key factor in defining synaptic function and plasticity (for review, see Cline, 2001; Scott and Luo, 2001; Jan and Jan, 2003). Molecular/genetic signaling determines this morphology and changing function by modulating microtubule-based dendritic shafts and actin-rich dendritic filopodia or spines (Fifkova and Delay, 1982; Matus et al., 1982; Micheva et al., 1998). Filopodia can serve multiple functions including acting as precursors to dendritic spines (Parnass et al., 2000 ) and pioneering the formation of dendritic arbors (Niell et al., 2004). Dendritic compartments undergo dynamic regulation and demonstrate both morphological and synaptic plasticity (Engert and Bonhoeffer, 1999; Maletic-Savatic et al., 1999; Toni et al., 1999; Yuste and Bonhoeffer, 2001). However, how these plasticity changes are regulated is poorly understood. We asked

\footnotetext{
Received Nov. 5, 2004; revised July 14, 2005; accepted Aug. 14, 2005.

This work was supported by awards to J.E.B. from the Searle Scholars Program and Whitehall Foundation for Neurobiology. We thank Dr. L. C. Griffith for generous gifts of CaMKII fly lines, Dr. D. Kiehart for the gift of the GMA fly line, and Dr. R. Fehon for various Moesin fly lines. We also thank Dr. H. Oda for actin:GFP lines, the Bloomington Stock Center, Dr. S. Ohsako for the anti-CaMKII antibody, and the lowa Hybridoma Bank for additional monoclonal antibodies. We thank members of the University of North Carolina Neuroscience Center, particularly Drs. Anthony LaMantia and Steve Crews, for helpful suggestions and comments. A multiphoton/confocal microscope imaging facility funded by P30NS45892 was used extensively.

Correspondence should be addressed to Jay E. Brenman, University of North Carolina at Chapel Hill, 105 Mason Farm Road, 8109A Neuroscience Research Building, Chapel Hill, NC 27599

DOI:10.1523/JNEUROSCI.2005-05.2005

Copyright $\odot 2005$ Society for Neuroscience $\quad$ 0270-6474/05/258878-11\$15.00/0
}

whether proteins thought to modulate the cytoskeleton could regulate the formation and stability of dendritic filopodia.

The Drosophila larval peripheral nervous system (PNS) contains external sensory, chordotonal, and multiple dendrite $(\mathrm{md})$ neurons (Bodmer and Jan, 1987). PNS neurons are postulated to transduce and relay multiple sensory inputs (Dethier, 1963; Bodmer and Jan, 1987). Different md neurons are postulated to preferentially transduce different sensory modalities consistent with distinct morphologies of md neuron subtypes (Grueber et al., 2003). Studies have demonstrated that $m d$ neurons can function during thermosensation (Liu et al., 2003; Tracey et al., 2003), mechanosensation (Grueber et al., 2001), and locomotor behavior (Ainsley et al., 2003). A subset of md neurons, dendritic arborization (da) neurons, are structurally reminiscent of most multipolar highly branched vertebrate neurons and provide a genetically tractable assay for identifying dendritic molecular regulators (Gao et al., 1999).

Calcium/calmodulin-dependent protein kinase II (CaMKII) is implicated as a regulator of both morphological and electrophysiological plasticity and therefore provides a molecular link between neural activation and downstream changes in plasticity (Silva et al., 1992a; Pettit et al., 1994; Wu and Cline, 1998; Zou and Cline, 1999). CaMKII is an abundant brain serine/threonine kinase ( $\sim 2 \%$ of total protein) (Erondu and Kennedy, 1985) represented by four distinct genes in mammals $(\alpha, \beta, \gamma, \delta)$ (for review, see Lisman et al., 2002). Autophosphorylation of threonine 286 [or Drosophila CaMKII (dCaMKII) threonine 287] produces an activated enzyme that no longer requires bound calci- 
um/calmodulin for kinase activity (Wang et al., 1998) (for review, see Lisman et al., 2002). We investigated whether these CaMKII protein variants, with distinct biochemical properties, could differentially influence dendritic structure.

We identify novel Drosophila dendritic phenotypes produced by CaMKII activity. Our results illustrate that Drosophila da neurons provide a genetically amenable system that can elucidate distinctions between cytoskeletal regulation and morphological regulation. This is the first study of fluorescence recovery after photobleaching (FRAP) and live-animal actin visualization applied to multipolar Drosophila sensory neurons. Activated CaMKII expression increases formation of dendritic filopodia, whereas decreasing stability and accelerating filamentous actin (F-actin) turnover in da dendrites. In parallel, we examined the effects of elevated Rac1, a Rho subfamily GTPase known for modulating cytoskeletal actin (Hall, 1998). Although activated CaMKII and Racl expression cause increases in filopodia formation and actin turnover in da neurons, these two proteins rely on different cellular machinery. CaMKII expression only affects neurons that normally contain filopodia, whereas Racl activity causes formation of dendritic filopodia more generally, without modulating structural stability. Our studies further demonstrate that phenotypic effects of CaMKII activity on dendritic structure are evolutionarily conserved (data herein) (Luo et al., 1996; Fink et al., 2003; Jourdain et al., 2003).

\section{Materials and Methods}

Fly stocks. We use guidelines from Flybase (www.flybase.org) for italicized gene names, as well as uppercase or lowercase designations. Proteins are nonitalicized. Fly strains used were Gal4 109(2)80 (Gao et al., 1999), UAS-GFP, UAS-myr::mRFP, UAS-Racl (all obtained from the Bloomington Stock Center, Bloomington, IN), UAS-actin::GFP (Verkhusha et al., 1999), UAS-GMA (Dutta et al., 2002), UAS-Nod::GFP, UAS-CaMKII, UAS-CaMKII T287A, and UAS-CaMKII T287D (Jin et al., 1998; Wang et al., 1998). Gal4 109(2)80 was recombined on the UASGFP ( $y w$; Gal4-109(2)80, UAS-GFP), the F-actin reporter UAS-GMA ( $y w$; Gal4-109(2)80, UAS-GMA), UAS-actin::GFP ( yw; Gal4-109(2)80, UAS-actin::GFP), and the membrane-targeted monomeric red fluorescent protein (mRFP) UAS-mry::mRFP ( $y w ;$ Gal4-109(2)80, $U A S-m y r:: m R F P)$ chromosomes using standard meiotic recombination. The Gal4 109(2)80, UAS-actin::GFP recombinant was chosen for most experiments for its prominent labeling of dendritic filopodia. We assessed the effect of CaMKII and Racl on Drosophila PNS neurons using UAS-CaMKII, UAS-CaMKII T287A, UAS-CaMKII T287D, and UASRac1. Recombinant chromosomes combining Gal4-109(2)80, UAS-actin::GFP and protein variant were made as follows: (1) Gal4 109(2)80, UAS-actin::GFP, UAS-CaMKII, (2) Gal4 109(2)80, UASactin::GFP, UAS-CaMKII T287A, (3) Gal4 109(2)80, UAS-actin::GFP, UAS-CaMKII T287D, and (4) Gal4 109(2)80, UAS-actin::GFP, UASRac1. Homozygous recombinant second instar larvae were used for all experiments unless otherwise indicated. All flies were maintained at $25^{\circ} \mathrm{C}$ in yeast-cornmeal vials.

Histochemistry. Second instar larvae were dissected while submersed in room-temperature 4\% paraformaldehyde in PBS. The anterior and posterior ends of the larvae were dissected and discarded to allow maximum penetration of fixative and antibodies to sensory neurons in abdominal segments. A single incision along the length of the dorsal midline was made to allow access to peripheral nervous system tissue. Dissected larvae were incubated in $4 \%$ paraformaldehyde/PBS for $1 \mathrm{~h}$ at room temperature and incubated for $30 \mathrm{~min}$ in blocking solution containing 5\% normal goat serum and $0.1 \%$ Triton in PBS (PBST). Antibody incubations were done in 5\% normal goat serum/PBST, and dilutions were the following: mouse monoclonal anti-dCaMKII, 1:25 (Takamatsu et al., 2003); rabbit anti-green fluorescent protein (anti-GFP), 1:1000 (Molecular Probes Eugene, OR); and mouse anti-Futsch, 1:200 (22C10; Iowa Hybridoma Bank, Iowa City, IA). The dissected larvae were incubated with primary antibodies overnight at $4^{\circ} \mathrm{C}$ and washed three times with PBST. Secondary antibodies, cyanine 2 (Cy2)-conjugated goat anti-rabbit (Jackson ImmunoResearch, West Grove, PA) and Cy3-conjugated goat anti-mouse (Jackson ImmunoResearch), were diluted 1:200 in 5\% normal goat serum/PBST and incubated with samples for $4 \mathrm{~h}$ at room temperature. Images were from abdominal hemi-segment A6, acquired under similar settings using a Zeiss (Oberkochen, Germany) LSM 510 confocal microscope with a $40 \times$ oil immersion lens. Briefly, system argon/helium-neon lasers were used to excite Cy2 and Cy3, respectively, and a $2 \mu \mathrm{m}$ optical slice was taken. To ensure that all filopodia emanating from the dendritic shaft were imaged, approximately eight $0.2 \mu \mathrm{m}$ optical slices were imaged as a Z-stack with a $4 \times$ digital zoom at $1024 \times 1024$ pixels. The Z-stack was then compiled into one image using the LSM 510 software. Images were sized and cropped with Adobe Photoshop and placed into Adobe Illustrator (Adobe Systems, San Jose, CA) for labels and arrangement.

Construction of Nod::GFP transgenic. The Nod:GFP fusion protein was made using a previously generated Nod:LacZ fusion construct (Clark et al., 1997). An EcoRI-KpnI fragment of enhanced GFP (Clontech, Mountain View, CA) was used to replace the corresponding fragment of LacZ in Nod::LacZ to create Nod::GFP. The Nod::GFP gene fragment was cloned into the pUAST vector (Brand and Perrimon, 1993). The transgenes were introduced into a w1118 stock by P-element-mediated transformation (Rubin and Spradling, 1982).

In vivo imaging. Homozygous recombinant second instar larvae were placed on an air-permeable membrane (cut-out $40 \mu \mathrm{m}$ cell strainer mesh; Falcon; BD Biosciences, Franklin Lakes, NJ) on a glass slide directly over a $1-\mathrm{cm}$-diameter hole to allow air diffusion. Larvae were covered in Halocarbon oil 27 (Sigma, St. Louis, MO) and gently coverslipped $(22 \times$ $50 \mathrm{~mm}$; Fisher Scientific, Pittsburgh, PA) to restrict movement but not cause bursting of the body wall. Confocal images of dendrite morphology were obtained with a Zeiss LSM 510 confocal microscope by exciting the $488 \mathrm{~nm}$ argon line to excite GFP. Abdominal hemi-segment A6 was imaged for all experiments. For filopodia counts (see Fig. 5A), the entire ddaA neuron dendritic arbor was imaged. Three micrometer Z-stacks (usually composed of 15 slices, each $0.2 \mu \mathrm{m}$ thick) at $1024 \times 1024$ pixels with a $20 \times$ lens were compiled to ensure that all filopodia protruding from the dendritic structure were imaged. Using the Zeiss LSM software, the ddaA neuron was traced from seven animals of each genotype, and all dendritic filopodia were classified by length and counted. Similar laser settings and Z-stacks were used for time-lapse experiments (see Fig. $5 B, C)$. Images were taken every $2-3 \mathrm{~min}$ for $15 \mathrm{~min}$ to ensure that filopodia did not emerge and retract during the $15 \mathrm{~min}$ and may have been missed. We measured the formation and disappearance of filopodia (see Fig. $5 B$ ) by tracing the entire $100 \mu \mathrm{m}$ length of the first ventral branch of the ddaA neuron and classifying filopodia as new if they formed after $t=$ 0 and disappeared if they were no longer visible during the $15 \mathrm{~min}$ interval. Seven ddaA dendritic lengths for each genotype were counted and plotted with Excel software (Microsoft, Redmond, WA). A $\chi^{2}$ test [calculated with SAS (Cary, NC) software] was used to determine differences in filopodia populations. To resolve the stability of a given filopodium over time (see Fig. 5C), 25 filopodia on the ddaA neuron (hemi-segment A6) were observed for $15 \mathrm{~min}$ at 2-3 min intervals. The Zeiss LSM software overlay function allowed tracing and measurements of these filopodia, which were categorized as elongating, retracting, or stable (within $0.2 \mu \mathrm{m}$ ) at $t=0$ and $t=15$, and averaged across seven animals of each genotype. The coverslip was removed, ensuring no harm to the specimen, and the larvae were placed in yeast-cornmeal vials. Only data from larvae that eclosed as adults $(>90 \%)$ were used.

Fluorescence recovery after photobleaching. Second instar larvae were mounted in halocarbon oil as described for in vivo imaging (above). Images were obtained using the confocal with a $488 \mathrm{~nm}$ argon line (Coherent, Santa Clara, CA) used to excite GFP. The argon line was set to $50 \%$ output, and the acousto-optical tunable filter (AOTF) slider was set at $0.5 \%$, minimizing unwanted photobleaching. The pinhole was adjusted to 5 Airy disks to obtain an optical section of $4 \mu \mathrm{m}$. Once a baseline time point was captured, filopodia were bleached by increasing the zoom $4 \times$ and drawing a region of interest (ROI) (Zeiss software) around a $\sim 2$ $\mu \mathrm{m}$ filopodium excluding the dendrite (see Fig. 6 A). At $50 \%$ output, the 
AOTF slider was increased to $100 \%$, and the ROI scanned for five pulses at $512 \times 512$ pixels $(\sim 3.0 \mathrm{~s})$, similar to previous FRAP studies (Star et al., 2002; Zito et al., 2004). After bleaching, the laser was reset to prebleach excitation for image capturing. Images were captured at $\sim 1$ min intervals for at least $12 \mathrm{~min}$. Only larvae that survived to eclosed adults $(\sim 90 \%)$, with filopodia that demonstrated recovery [similar to Zito et al. (2004)] ( $~ 80 \%$ of total) and stayed morphologically similar (within 10\% length), were used for quantification. In addition, stability of filopodium was not significantly different between low level and FRAP laser excitation (data not shown). One filopodium on the ddaA neuron from seven individual animals was quantified for FRAP. Images from FRAP experiments were analyzed by NIH ImageJ software. Filopodium were traced, an average intensity of fluorescence calculated, background fluorescence subtracted, and normalized to prebleach levels. FRAP curve was fit based on an equation $\left(F(t)=1-f_{\mathrm{s}}-f_{\mathrm{f}} \mathrm{e}^{-t / \lambda}\right)$ derived by Star et al. (2002), and $t_{1 / 2}$ was calculated by Kaleidagraph software (Synergy Software, Reading, PA).

\section{Results}

An assay system for cytoskeletal and dendritic analysis

We used the Gal4/UAS system (Brand and Perrimon, 1993) to express fluorescently tagged proteins, assessing the dendritic compartmentalization and structure of $\mathrm{da}$ neurons. The GAL4 109(2)80 enhancer line allows clear visualization of da neuron morphology, is highly specific to postmitotic md sensory neurons, and labels all six da neurons (ddaA-F), one bd neuron, and one tracheal dendrite neuron in the 12 neurons of the dorsal cluster (Gao et al., 1999). These neurons have been classified (I-IV) based on the complexity of the dendritic arbor (Grueber et al., 2003). Previously, we observed dendritic protrusions in larvae faintly visible with GFP but unlabeled by Tau::GFP (Gao et al., 1999). We hypothesized these protrusions would be actin-enriched, similar to mammalian dendritic filopodia and spines (Fifkova and Delay, 1982; Matus et al., 1982). Indeed, actin:GFP fusion protein $(\beta$ Actin5C) expressed in da neurons allows visualization of numerous finger-like dendritic filopodia (Fig. $1 B$ ), more intensely labeled than with GFP alone (Fig. $1 A$ ). Although GFP is not completely excluded from filopodia (Fig. $1 A$, inset), the small volume of dendritic filopodia results in decreased fluorescence compared with actin: GFP, which is specifically targeted to filopodia (Fig. $1 \mathrm{~B}$, inset). GMA (Dutta et al., 2002), a chimeric GFP construct containing 140 amino acids of the F-actin-binding protein Moesin, was expressed in transgenic animals to further characterize these filopodia (Fig. 1C$E$ ). Both GMA (Fig. 1C-E) and rhodamine-phalloidin (data not shown) labeling gave qualitatively similar results with strong enrichment in filopodia. Furthermore, GMA has been demonstrated to $\mu \mathrm{m} ; \boldsymbol{C}, \boldsymbol{D}, \boldsymbol{F}, 5 \mu \mathrm{m}$
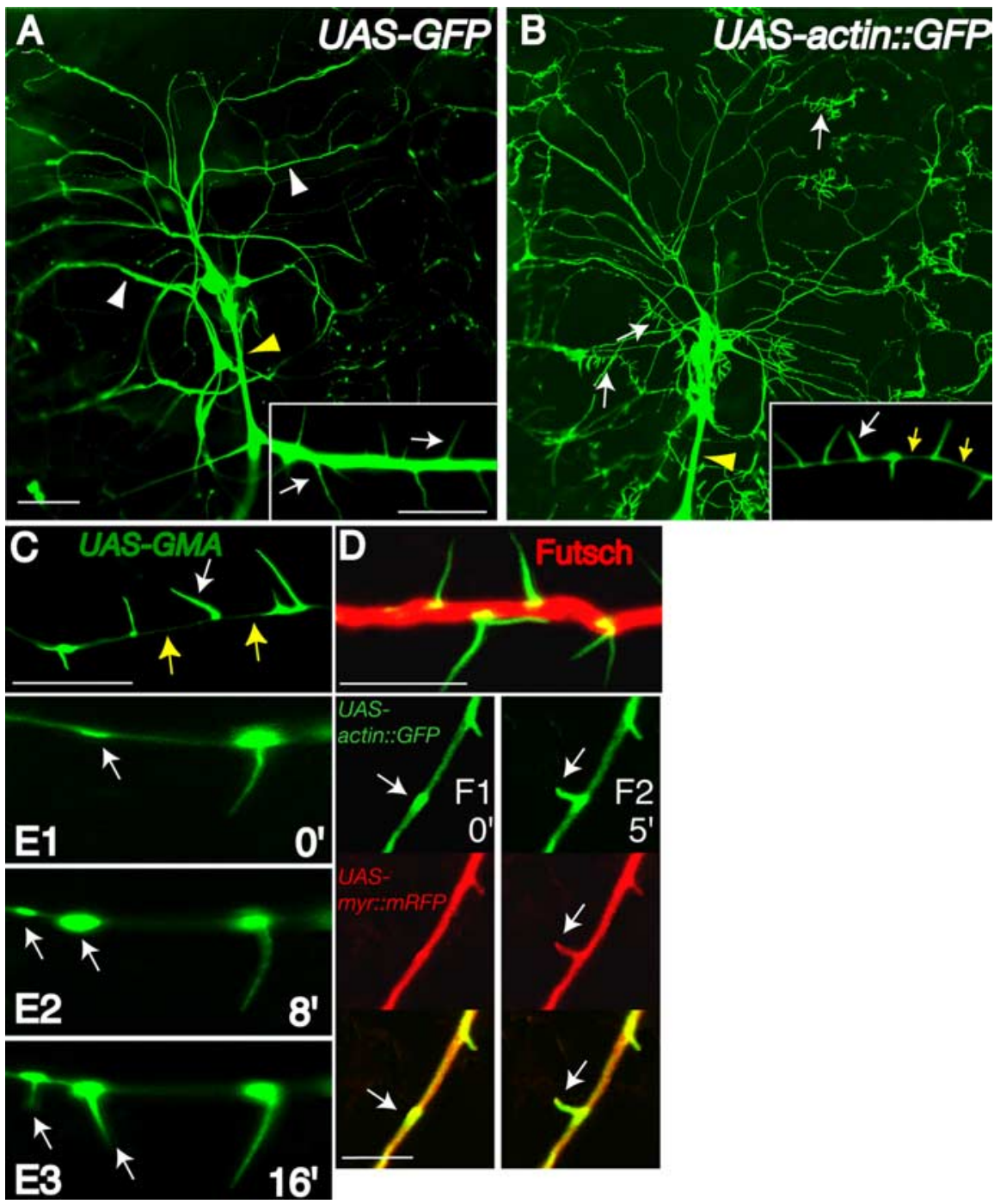

Figure 1. Drosophila da neurons contain actin-rich filopodia restricted to dendrite compartments. Single-neuron dendrite images $(\boldsymbol{A}-\boldsymbol{F})$ are from the ddaA neuron from the dorsal cluster of sensory neurons. All images throughout this study contain hemi-segment A6 dorsal cluster da neurons with anterior toward the left and dorsal toward the top. $A$, A second instar larva ( $y w$; Gal4-109(2)80, UAS-GFP) expressing GFP in da neurons demonstrates strong dendritic shaft (white arrowheads) and axon fascicle (yellow arrowhead) labeling. $\boldsymbol{B}$, In comparison, a second instar larva ( $y w$; Gal4-109(2)80, UAS-actin::GFP) expressing actin::GFP reveals actin-rich dendritic filopodia along dendrites (white arrows) that are absent on axonal shafts (yellow arrowhead). Actin::GFP demonstrates strong enrichment in dendritic filopodia with only limited fluorescence in dendritic shafts (inset, yellow arrows). C, When a second instar larva ( yw; Gal4-109(2)80, UAS-GMA) expresses GMA, an F-actin-binding GFP fusion protein, dendritic filopodia are strongly labeled with faint localization in dendritic shafts (yellow arrows). D, A transgenic second instar larva expressing GMA immunolabeled against Futsch (red), a microtubule-binding protein, GMA ( $\alpha$-GFP; green), reveals mostly separate dendritic localization for microtubule- and actin-associated proteins. E1-E3, In vivo 8 min time-lapse images of a GMA expressing transgenic second instar larva indicate early recruitment of F-actin to dendritic patches that predict sites of future dendritic filopodia outgrowth (arrows) (time in minutes). F1, F2, In vivo 5 min time-lapse images of a second instar larva ( $y w$; Gal4-109(2)80, UAS-actin::GFP, UAS-myr::mRFP) expressing actin:GFP (green; arrows) and myr::mRFP reveal membrane outgrowth (red; arrows) is closely associated with actin::GFP outgrowth (green; arrows). Scale bars: (in $\boldsymbol{A}) \boldsymbol{A}, \boldsymbol{B}, 50 \mu \mathrm{m}$; insets, 5

give qualitatively similar staining as rhodamine-phalloidin in other cell types as well (Dutta et al., 2002). However, numerous in vivo microtubule reporters, including Tau::GFP, which binds microtubules in vivo (Brand, 1995), and tubulin::GFP (Grieder et al., 2000) failed to label dendritic filopodia (data not shown). Immunolabeling against Futsch, a microtubule-associated protein 1B-like protein, which binds microtubules (Hummel et al., 2000) (Fig. 1D), also failed to label these filopodia, suggesting these structures are devoid of microtubules.

Observing dendritic filopodia development in real time al- 

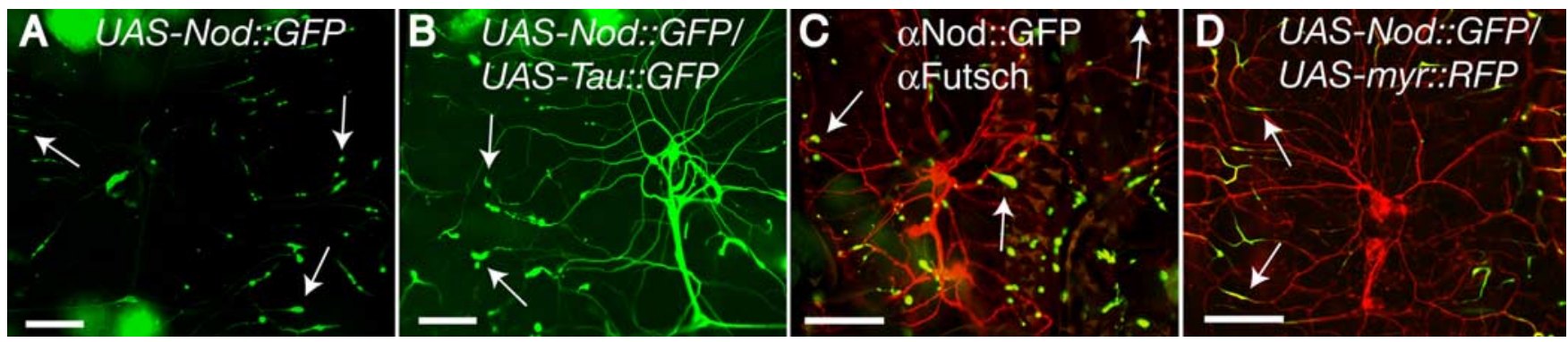

Figure 2. Asynaptic Drosophila da neurons contain minus-end-out microtubule arrays in dendrites. A, A second instar larva ( $y w$; Gal4 109(2)80, UAS-Nod:GFP) expressing Nod:GFP, a minus-end reporter for microtubules, demonstrates Nod:GFP enrichment at the tips of da dendrites (arrows). B, A second instar larva ( yw; Gal4 -109(2)80, UAS-Nod::GFP/Gal4 -109(2)80 UAS-Tau::GFP) heterozygous for both Tau::GFP, a microtubule-binding protein that labels dendrites, and Nod::GFP demonstrates that Nod::GFP accumulates at the ends of dendrites collinear with the dendrite shaft. C, A second instar larva ( $y w$; Gal4 109(2)80, UAS-Nod::GFP) immunolabeled against Nod::GFP ( $\alpha$ GFP; green) and Futsch ( $\alpha$ Futsch; red) reveals colabeling (yellow) as puncta at the ends of da neuronal dendrites (arrows). D, A second instar larva ( $y w$; Gal4 109(2)80 UAS-myr::RFP/UAS-Nod::GFP) heterozygous for both myr:RFP and Nod::GFP demonstrates Nod:GFP accumulation at the ends of da dendrites (arrows). Scale bars, $50 \mu \mathrm{m}$.

lows the discrimination of subtle, but distinct, steps in filopodia development and F-actin regulation. Whole-animal time-lapse imaging demonstrates that F-actin-rich patches accumulate in the dendrite and premark the directed site of future dendritic filopodia emergence (Fig. $1 E$ ). In time-lapse analyses, every emerging filopodium is preceded by a GMA accumulation ( $n=30$ filopodia). We hypothesized these actin-based outgrowths resulted from de novo protrusion and not merely sudden actin transport into preformed membranous structures. We used transgenic animals coexpressing membrane-targeted, myristoylated monomeric red fluorescent protein (myr::mRFP) and actin::GFP fusion protein. Every single protrusion labeled with myr:mRFP, representing all stages of outgrowth, was always closely colabeled with actin: GFP in live animals (Fig. $1 \mathrm{~F}$ and data not shown). These data suggests we were observing actin-coupled filopodia outgrowth and not sudden actin-transport events.

Whereas mammalian peripheral sensory endings are predominantly axon-like, Drosophila peripheral sensory endings are believed to be dendritic-like compartments (Bodmer and Jan, 1987). da sensory dendrites do not receive axonal synapses but share similarities with vertebrate dendrite structure including filopodia on dendritic but not axonal shafts (Fig. $1 B$ ). We examined microtubule orientation in vivo to further examine whether cytoskeletal architecture of da neurons reflects the same architecture as dendrites that receive synapses. A hallmark of mammalian dendrites is the presence of mixed populations of microtubules in dendritic compartments (Baas et al., 1988). Drosophila synaptic CNS mushroom body neurons display concentrated Nod::LacZ fusion protein at the distal tips of dendrites. In all analyzed cell types, Nod::LacZ functions as a minus-end microtubule reporter (Clark et al., 1997). We used this Nod::LacZ construct to generate a Nod::GFP fusion protein to conduct live-animal imaging for da dendrite analysis. Nod::GFP expressed in da sensory neurons enriches at the distal tips of da neuron dendrites (Fig. 2), indicating a similar microtubule architecture with mushroom body dendrites, which do receive axonal inputs in the adult brain. Although it is not known how these reporters would localize in mammalian neurons, this reflects a Drosophila dendrite microtubule array (Lee et al., 2000) and is consistent with minus-end-out microtubules. A summary of fusion proteins used in this study and their localizations is provided in Table 1 (available at www. jneurosci.org as supplemental material).
CaMKII can potently regulate Drosophila dendritic filopodia Structural similarities between Drosophila da dendrites and vertebrate dendrites prompted us to investigate whether functional regulation could be similar as well. CaMKII, present in da sensory neurons (see Fig. 5, inset) (Takamatsu et al., 2003), has been demonstrated to affect dendritic morphology (Cline, 2001) as well as filopodia and spine formation (Jourdain et al., 2003). We expressed CaMKII protein variants (Jin et al., 1998) to evaluate potential roles for CaMKII regulation of dendritic morphology. Wild-type CaMKII (UAS-CaMKII), or single point mutant versions in the autoregulatory domain, threonine 287 (UASCaMKII T287D, UAS-CaMKII T287A), were driven by the same promoter element, Gal4 109(2)80. To enable live-animal imaging, transgenic larvae bearing the Gal4 109(2)80 and UAS-actin::GFP element were used for the remaining experiments. The CaMKII T287D mutation is a phosphomimetic version of phospho-threonine that renders the enzyme constitutively active without requiring bound calcium/calmodulin. In contrast, the CaMKII T287A mutation allows the enzyme to become active in the presence of calcium/calmodulin but loses the ability to become persistently active when calcium levels fall (Fong et al., 1989; Waldmann et al., 1990; Wang et al., 1998).

There were no detectable differences of dendrite structure between wild-type (Fig. $3 A, F, K)$ and wild-type UAS-CaMKII transgenic larvae (Fig. $3 B, G, L$ ). However, significant increases in dendritic filopodia density were observed in class III neurons expressing activated CaMKII T287D (Fig. 3D,N). The CaMKII T287D phenotype becomes most obvious when comparing equivalent dendrite branches from the same neurons in wildtype, UAS-CaMKII, UAS-CaMKII T287A, and UAS-CaMKII T287D larvae (Fig. $3 K-N$ ). We counted all dendritic filopodia on the entire ddaA dendritic arbor for second instar larvae bearing CaMKII transgenes. We further classified these filopodia based on length to detect any dramatic differences in morphology. The UAS-CaMKII T287D phenotype was confined to the dendritic compartment, because no formation of filopodia on axon shafts in CaMKII T287D animals occurred (Fig. 3N, yellow arrowhead). The CaMKII T287D phenotype demonstrates a more severe phenotype with increasing transgene number. Increasing CaMKII activity, by increasing CaMKII T287D transgene number, increased the number of filopodia formed without substantially changing the length/number distribution (Fig. $4 A$ ). In contrast, even two transgenes of CaMKII T287A or wild-type CaMKII had no effect on total numbers of filopodia formed (Fig. $4 A$ ). CaMKII 

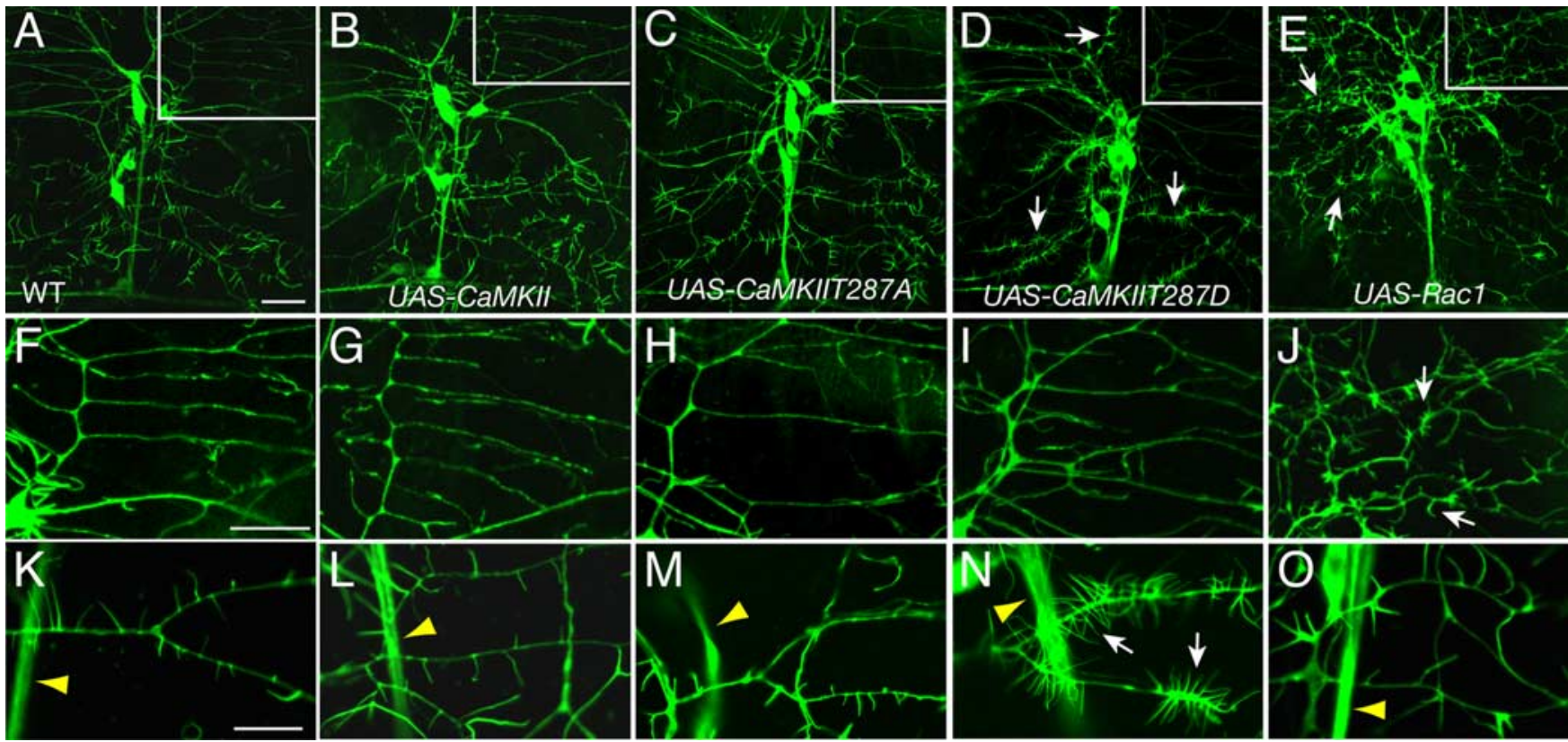

Figure 3. CaMKII T287D expression in transgenic larvae induces high densities of filopodia on class III neuronal dendrites, whereas Rac1 expression induces filopodia formation on non-class III da neuronal dendrites. All larvae are second instars, homozygous for Gal4 -109(2)80, UAS-actin::GFP and UAS-CaMKII variants or UAS-Rac1 transgenes. $A, F, K$, Dendrites from a wild-type (WT) larva are indistinguishable from a transgenic larva expressing wild-type CaMKII $(B, G, L)$ and nearly identical to a larva expressing CaMKII T287A $(C, H, M) \cdot D, I, N$, In contrast, a transgenic larva expressing CaMKII T287D demonstrates striking increases in numbers of filopodia on class III da neuronal dendrites (arrows). $\boldsymbol{E}, \mathbf{J}, \mathbf{O}$, Rac1 expression in a transgenic larva causes increased dendritic branching and filopodia formation on all da neurons. $\boldsymbol{F}-\boldsymbol{J}$, Boxed regions (above, $\boldsymbol{A}-\boldsymbol{E}$ ) reveal that a class I da neuron, ddaE, does not have filopodia when expressing any protein variant except Rac1 ( $\boldsymbol{J}$, arrows). $\boldsymbol{K}-\mathbf{0}$, Equivalent dendritic branches from the class III ddaA neuron have marked increases in dendritic filopodia when expressing CaMKII T287D (N) compared with WT (K), CaMKII (L), CaMKII T287A $(\boldsymbol{M})$, or Rac1 (0). Note that axon shaft fascicles (yellow arrowheads) in all genotypes lack filopodia. Scale bars: (in $\boldsymbol{A}, \boldsymbol{F}) \boldsymbol{A}-\boldsymbol{F}, 20 \mu \mathrm{m}$; (in $\boldsymbol{F}$ ) $\boldsymbol{F}-\boldsymbol{J}, 10 \mu \mathrm{m}$; (in $\boldsymbol{K}) \boldsymbol{K}-\mathbf{0}, 10 \mu \mathrm{m}$. All larvae for the remaining figure legends are of the background genotype yw; Gal4 109(2)80, UAS-actin:GFP to allow visualization of dendritic filopodia.

T287A did produce significantly longer filopodia $(>10 \mu \mathrm{m})$ and fewer short filopodia $(<2 \mu \mathrm{m})$ than wild-type CaMKII $\left(\chi^{2}\right.$ test; $p<0.0001$ ) (Fig. 4A).

Increased filopodia formation appears highly specific to CaMKII T287D and was not observed with other kinases and actin modulatory proteins, including Gal4/UAS expression of Rho kinase (Winter et al., 2001), as well as active and inactive versions of actin modulatory proteins such as Moesin (data not shown). Although Cut protein is known to alter cell fates of subtypes of da neurons (Grueber et al., 2003), expression of this homeodomain protein did not increase filopodia formation on class III neurons but simply converted other neurons into filopodia-bearing neurons (data not shown) (Grueber et al., 2003). Thus, CaMKII expression does not affect cell fate as Cut expression does but uses signaling mechanisms that specifically regulate the actin cytoskeleton.

\section{Rac1 and CaMKII expression have distinct morphological phenotypes}

We examined a known modifier of the actin cytoskeleton and dendritic structure, Rac1, to further explore specificity of the CaMKII T287D phenotype. In Drosophila, two groups have demonstrated a genetic requirement for $\mathrm{racl}$ in da neurons. Loss of racl function results in decreased dendritic branching (Lee et al., 2003), whereas increased wild-type Racl expression results in increased branching (Lee et al., 2003; Emoto et al., 2004). Furthermore, dominant-negative Racl suppresses an increased branching phenotype produced by Furry kinase loss-of-function mutants (Emoto et al., 2004). When we expressed wild-type Rac1 using Gal4 109(2)80, we observed dramatic increases in dendritic branching (Fig. $3 E, J$ ), as reported previously (Lee et al., 2003; Emoto et al., 2004). We also observed actin::GFP-containing filopodia on da neuron classes that normally lack filopodia (Fig. $3 J)$. In contrast, the CaMKII T287D phenotype was confined to class III neurons and did not create filopodia de novo in neurons that normally lack abundant filopodia (Fig. $3 D, I$ ). Densities of spines and filopodia also vary characteristically among different mammalian neuronal types, and not all vertebrate dendrites have filopodia or spines. Racl expression leads to more filopodia on transgenic larvae than wild-type or UAS-CaMKII larvae solely by increasing dendritic branching and forming filopodia on normally nonfilopodia bearing neurons (Fig. 3) without altering filopodia density (Fig. $3 \mathrm{~K}, \mathrm{O}$ ). These observations suggest distinct mechanisms for CaMKII- and Racl-mediated filopodia regulation.

\section{Activated CaMKII, but not Rac1, increases the dynamic} nature of dendritic filopodia

We compared the effects of CaMKII and Rac1 signaling on dendrite stability. The ability to repeatedly image equivalent neurons between different transgenic animals over time allows for direct comparisons of dendritic dynamics. In mammals, dendritic filopodia and dendritic spines are spontaneously dynamic, such that only a small percentage of spines persist after a few days (Trachtenberg et al., 2002). Drosophila dendritic filopodia are also intrinsically dynamic (Fig. $4 B, C$ ). Identical $100 \mu \mathrm{m}$ dendrite segments of the class III neuron ddaA were imaged over a $15 \mathrm{~min}$ interval in wild-type, wild-type CaMKII-, CaMKII T287D-, CaMKII T287A-, and Rac1-expressing larvae (Fig. 4 B, C). Each filopodium was monitored continuously for its stability over time and whether filopodia formed or disappeared on the dendritic segment. Time-lapse analysis revealed that CaMKII T287D dramatically increases the numbers of newly formed and disappearing filopodia during development (Fig. 4B). More filopodia 

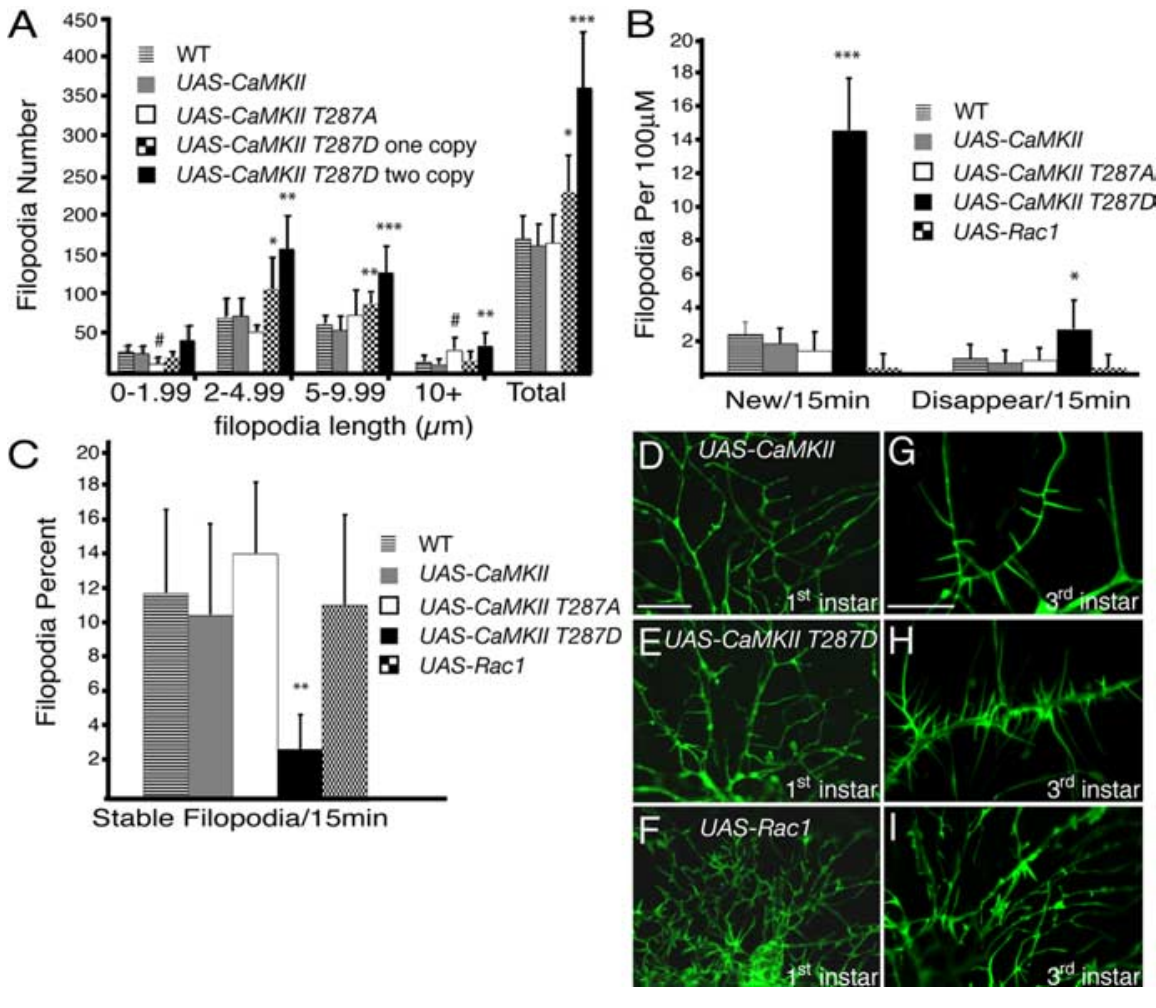

Figure 4. Class III dendrites of transgenic larvae expressing CaMKII T287D contain increasing filopodia in a gene-copydependent manner, whereas CaMKIII T287D activity, but not Rac1 activity, modulates dendritic filopodia stability. A, Second instar larvae expressing CaMKII T287D possess increasing numbers of filopodia on ddaA neurons with increasing transgene number. Expression of CaMKII T287A shifts the distribution toward slightly longer filopodia ( ${ }^{\#} p<0.0001 ; \chi^{2}$ test; $n=7$ of wild-type CaMKII and CaMKII T287A) but does not increase total number $\left({ }^{*} p<0.06 ;{ }^{* *} p<0.006 ;{ }^{* * *} p<0.0007 ; t\right.$ test; $n=7$ of each genotype; all filopodia from the entire ddaA dendritic arbor were counted.) $\boldsymbol{B}$, Time-lapse imaging (2-3 min intervals; total, 15 $\mathrm{min}$ ) of the ventral dendrite segment $(100 \mu \mathrm{m})$ of neuron ddaA demonstrates that CaMKII T287D, but not Rac1, activity increases formation and disappearance of filopodia $\left({ }^{*} p<0.02{ }^{* * * *} p<8 \times 10^{-6}\right)$. C, CaMKIl expression decreases the stable pool of filopodia when compared with WT, WT CaMKII, CaMKII T287A, and Rac1 $\left[{ }^{* *} p<0.004 ; n=7 ; 25\right.$ filopodia from a $100 \mu$ m ventral dendrite segment of neuron ddaA segment (similar to $\boldsymbol{B}$ ) were imaged every $2-3 \mathrm{~min}$ for $15 \mathrm{~min}$. The filopodia were then classified as stable, elongating/retracting, or disappeared. Seven animals for each genotype were quantified]. Error bars represent SEM. $\boldsymbol{D}-\boldsymbol{F}$, Newly hatched first instar larvae and third instar larvae $(\boldsymbol{G}-\boldsymbol{I})$ reveal timing differences between CaMKII T287D and Rac1 mutant phenotypes. WT CaMKII (D) and CaMKII T287D $(\boldsymbol{E})$ first instar larvae appear similar. In contrast, a Rac1 ( $\boldsymbol{F})$-expressing larva shows an early branching and filopodia mutant phenotype. G-I, Third instar larvae class III neuron ddaF demonstrates CaMKII T287D expression has a cumulative phenotype $(\boldsymbol{H})$, consistent with continuous increased filopodia formation $(\boldsymbol{B})$. The Rac1 phenotype appears relatively stable, with increased branching most prominent. Scale bars: $\mathbf{D}, \mathbf{G}, 10 \mu \mathrm{m}$. WT, Wild type.

are formed than disappear, explaining why CaMKII T287D animals have such a large increase in filopodia that becomes more pronounced over time (Fig. 4B,E,H). In contrast, neither Rac1 nor nonactivated versions of CaMKII had such a phenotype (Fig. $4 B, D-I)$. Although fractions of elongating and retracting filopodia are not significantly different between different genotypes (data not shown), there are significantly fewer stable filopodia in CaMKII T287D larvae than in CaMKII-, CaMKII T287A-, or Rac1-expressing larvae (Fig. 4C). Racl appears to preferentially modulate filopodia formation, whereas CaMKII appears to modulate structural dynamics. Observing different developmental stages supports the time-lapse experiments. Whereas the Rac1 branching phenotype is readily apparent early in newly hatched first instar larvae (Fig. $4 F$ ), the CaMKII T287D phenotype does not become readily apparent until the second instar stage (Fig. $3 N$ ), becoming even more prominent later in development (Fig. $4 H$ ), demonstrating a cumulative phenotype in CaMKII T287D mutants (Fig. $4 B-I$ ).

We explored whether phenotypic differences for CaMKII variants would be associated with corresponding protein local- ization differences in dendrites. Mammalian CaMKII- $\alpha$, which is most similar to Drosophila CaMKII, is abundant in somatodendritic compartments. Drosophila CaMKII is found in somatodendritic compartments (Takamatsu et al., 2003) but shows strong protein enrichment at F-actin-rich sites, particularly at the base of dendritic filopodia in second instar larvae (Fig. 5). Filopodia are almost always associated with immunoreactive nodes in dendrites (Fig. 5). CaMKII protein variants do not appear to show gross subcellular localization differences, as examined in fixed tissue during a single time point. Activated CaMKII T287D does result in a slight swelling of dendritic cytoplasm, particularly where dense clusters of filopodia form (Fig. 5C). However, microtubule compartment diameter appears unchanged as assessed by either 22C10 (antiFutsch) immunostaining or tubulin::GFP expression (data not shown).

\section{CaMKII and Racl increase actin turnover in dendrites}

We further explored how CaMKII and Racl might modulate actin turnover within dendritic filopodia by examining actin dynamics in living larvae. A previous study using dissociated hippocampal neurons expressing actin::GFP has demonstrated rapid turnover of F-actin in dendritic spines. Actin turnover in spines occurs on the seconds to minute time scale, although the spines themselves can be semistable (Star et al., 2002). We used a similar actin::GFP construct but conducted fluorescent recovery after FRAP studies using intact second instar Drosophila larvae. da neurons are ideally suited for FRAP analysis. These neurons are attached to a single layer of optically transparent epidermal cells and allow localized photobleaching of a single filopodium (Fig. 6). Such FRAP studies are particularly powerful with Drosophila because pathways can be modulated genetically, instead of pharmacologically, and the location of these neurons allows noninvasive experimentation in intact animals. FRAP analysis was conducted with low laser settings and high detector gain for data acquisition and high-power focused pulses for photobleaching (Fig. 6A) (Star et al., 2002). Photobleaching itself did not cause retraction of filopodia (Fig. 6A). Furthermore, FRAP data were only used from larvae that could later eclose to adults ( $\sim 90 \%)$. Similar to the mammalian studies, we observed rapid turnover of actin::GFP within dendritic filopodia but on a slightly slower time scale than mammals (data herein) (Fig. 6B) (Star et al., 2002). In addition, fluorescent recovery was usually observed at the distal filopodia tip first (data not shown), consistent with addition of new monomer at the plus end.

Larvae expressing activated CaMKII T287D show a dramatic increase in the postdiffusion turnover of actin::GFP with a clear statistically significant shift relative to either wild-type CaMKII or T287A transgenic animals (Fig. 6B). Rac1, like CaMKII 

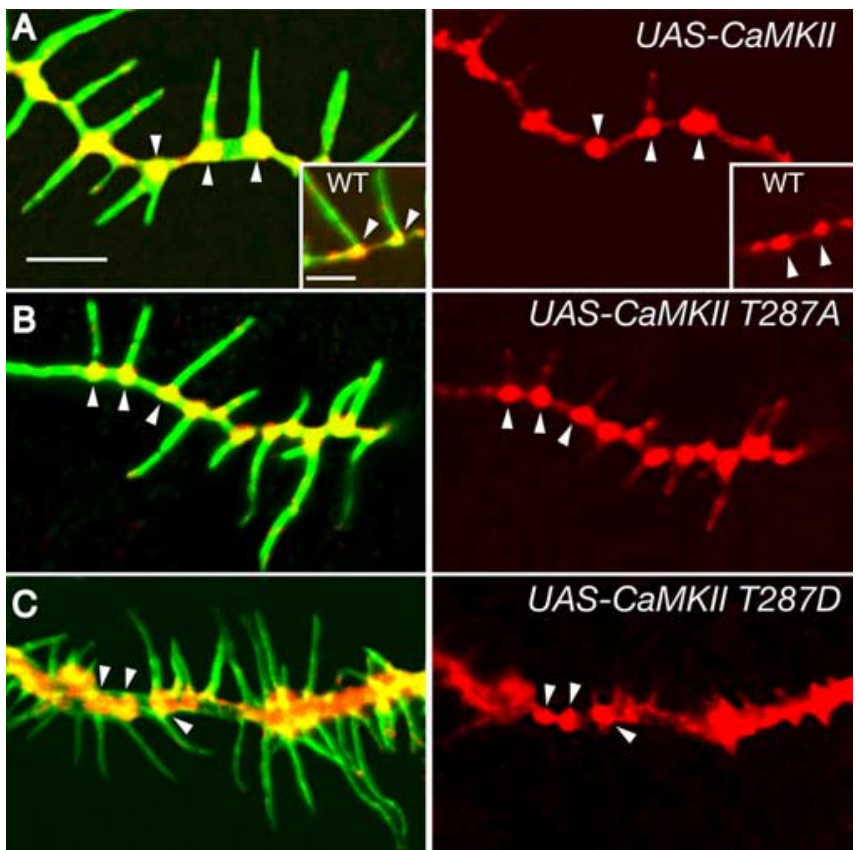

Figure 5. CaMKII protein variants show enrichment at the intersection of F-actin and microtubule compartments with similar dendritic subcellular localization in fixed specimens. $\boldsymbol{A}-\boldsymbol{C}$, Anti-CaMKII immunolabeling (red) of endogenous CaMKII ( $\boldsymbol{A}$, inset) expressed wild-type (WT) CaMKII (A), CaMKII T287A ( $\boldsymbol{B})$, or CaMKII T287D ( $\boldsymbol{C}$ ) in second instar larvae of neuron ddaA. CaMKIl enrichment (arrowheads) is found at the intersection of dendrites and filopodia (antiGFP; green projections). Note the phenotype in CaMKII T287D ( $\boldsymbol{C}$ ) and the association of individual filopodia with CaMKII-immunopositive clusters in all larvae. Scale bars: $\boldsymbol{A}, 5 \mu \mathrm{m}$.

T287D, also increased actin turnover (Fig. 6B), although effects on morphological stability are significantly different (Fig. $4 B, C$ ), suggesting dendrite structure and the cytoskeleton within it, can be independently regulated. We only evaluated filopodia that eventually recover, with amounts of fluorescence recovery plateauing at similar levels, $>80 \%$ of prebleach levels (Fig. $6 \mathrm{~B}$ ), similar to previous methodology for mammalian neurons (Star et al., 2002). The longer time frame for fluorescent recovery in wildtype CaMKII or CaMKII T287A larvae resulted in more heterogeneous data between larvae of the same genotype. This phenomenon possibly reflects the structurally dynamic nature of dendritic filopodia, which over longer time periods would allow for more changes in actin regulation. Conversely, individual filopodium themselves could be heterogeneous, with different filopodium receiving various signals affecting actin dynamics.

\section{Discussion}

\section{An assay for genetic analysis of dendrite structure} and cytoskeleton

Developing forward genetic assays for neurite development, particularly in whole animals, presents unique challenges. Dendrites, in particular, branch extensively, travel short distances, and display much more structural heterogeneity than axons. In addition, dendrites in the CNS exist in dense neuropil that require labeling neuronal subsets to clearly identify specific dendritic arbors for visual screening. It is for this reason that we analyze physically separated Drosophila PNS neurons instead. Drosophila sensory dendrites are large and traverse large distances to innervate extensive epidermal surface area. This large territorial coverage and physical separation result in dendritic structure with highresolution visual clarity compared with CNS neurons. Although by no means an identical comparison to CNS dendrites, some crude aspects of Drosophila PNS and CNS dendrites are similar. Drosophila da neurons provide a unique opportunity to develop genetic assays for dendrite development entirely in vivo. Our studies exploit these benefits to identify novel phenotypes for CaMKII and Rac1 signaling in Drosophila.

Drosophila da neuron dendrites also share biochemical similarity with Drosophila CNS dendrites. da neurons display minusend-out microtubule arrays (Fig. 2) found uniquely in dendrites. Developing neurons display a distal minus-end-out array in dendrites, a key event in dendrite versus axon differentiation (Yu et al., 2000). In contrast, axonal compartments have solely ( - ) to $(+)$ microtubule arrays from cell body to axon terminal (Baas et al., 1988; Burton, 1988). Although da dendrites do not receive axonal contacts, Nod reporters demonstrate similar enrichment at the tips of dendrites in both da sensory and mushroom body (CNS) axonally innervated neurons (Lee et al., 2000). da sensory neurons also have distinct actin-rich dendritic compartments, similar to dendrites in the Drosophila brain (Scott et al., 2003). Soluble GFP weakly labels filopodia when compared with actin::GFP or GMA, which target GFP specifically to filopodia. These labeling patterns are similar with mammalian neurites wherein GFP is a volumetric indicator in neurites that intensely labels dendrites, with weak labeling of spine necks of small diameter $(\sim 0.1 \mu \mathrm{m})$ (Harris and Kater, 1994; Sabatini et al., 2002).

FRAP analysis of actin-rich dendrite compartments in mammals demonstrates a fast ( <2 s) (Star et al., 2002; Zito et al., 2004) diffusion-limited FRAP recovery stage followed by a slower, longer recovery stage. We obtained similar FRAP results whereby GFP recovery in dendrite shafts or filopodia occurs within seconds (data not shown), whereas actin::GFP FRAP recovery demonstrates a longer, slower time frame (Fig. 6). This postdiffusion, longer recovery stage is inferred to reflect dynamic and mobile pools of actin (Star et al., 2002; Zito et al., 2004). Actual measurements of globular actin and F-actin, however, require biochemical-based analysis. The actin::GFP used in our studies behaves similarly to actin:GFP in mammalian studies, except the small size of Drosophila larvae make them amenable to wholeanimal unanesthetized studies. Furthermore, we demonstrate that the effects of genetic perturbations on cytoskeletal turnover can be analyzed in intact transgenic animals with FRAP, providing cytoskeletal information to link molecules to phenotypes.

\section{CaMKII regulates dendritic structural plasticity}

CaMKII, which is expressed in Drosophila da neurons (Fig. 5), is commonly suggested to link neural activation with functional modification of circuits. In mouse models, altered expression of CaMKII- $\alpha$ leads to abnormal behavior (Chen et al., 1994), altered LTP in the hippocampus (Silva et al., 1992a; Pettit et al., 1994), reduced experience-dependent plasticity in barrel cortex (Glazewski et al., 1996), and impaired spatial learning (Silva et al., 1992b). How CaMKII modulates the cytoskeleton to bring about these changes is primarily unknown. Two groups have shown that CaMKII activity can alter filopodia formation in mammals (Fink et al., 2003; Jourdain et al., 2003). We demonstrate, with intact animals in real time, that CaMKII signaling affects dendritic stability by altering cytoskeletal and structural dynamics.

Similar morphological effects of CaMKII signaling may reflect the highly conserved amino acid sequence and biochemical regulatory mechanisms shared between CaMKII in mammals and Drosophila (Wang et al., 1998). A version of CaMKII, T287A, which can still be activated but not autophosphorylated, appears to slightly shift the distribution of filopodia, such that neurons expressing T287A CaMKII have slightly longer $(>10 \mu \mathrm{m})$, and 


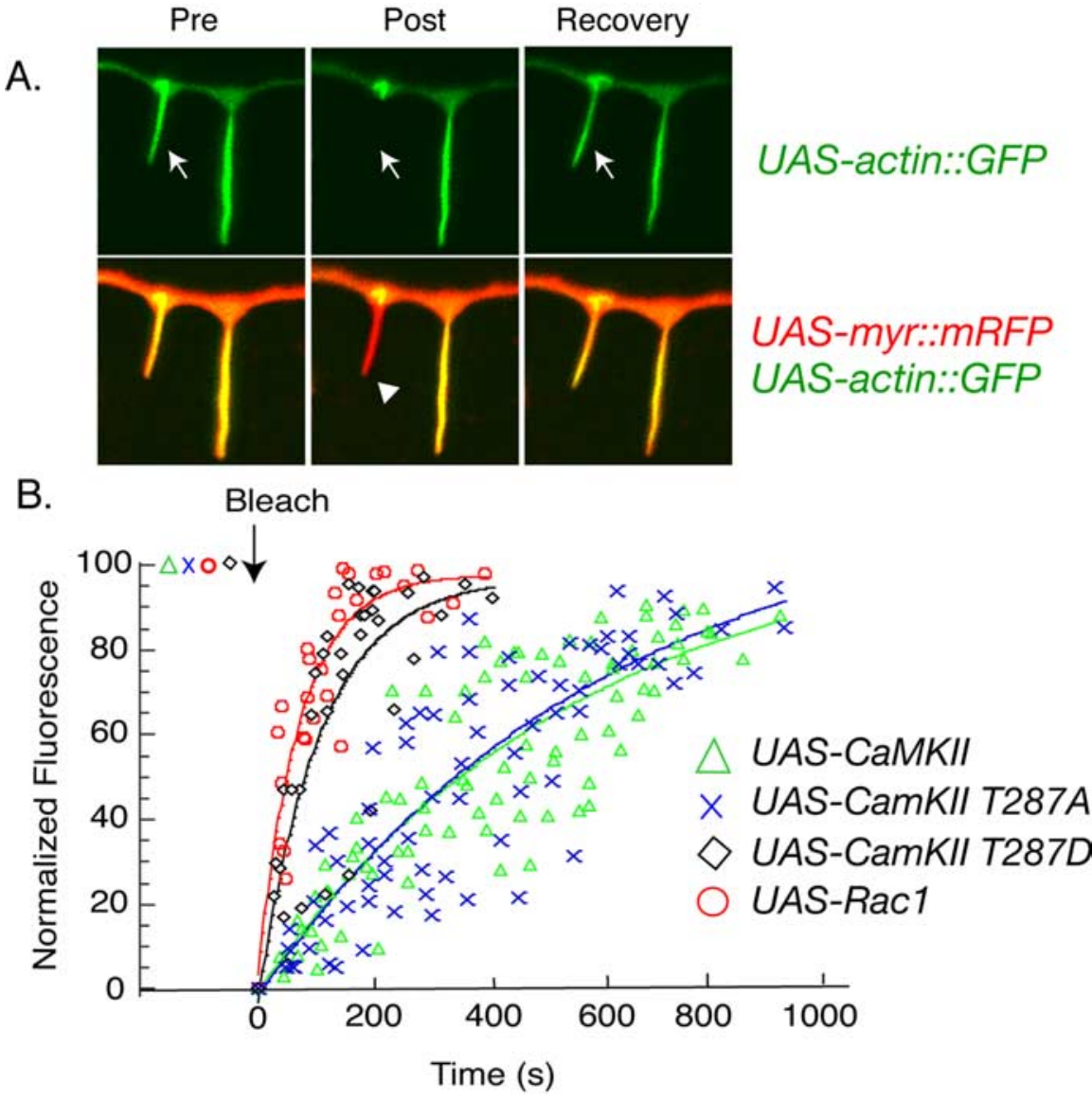

Figure 6. Intact CaMKII T287D and wild-type Rac1 transgenic larvae demonstrate significantly increased actin::GFP turnover in dendritic filopodia. A, Actin::GFP turnover in dendritic filopodia was demonstrated using fluorescence recovery after FRAP (arrows). A transgenic animal containing both UAS-actin::GFP and membrane-targeted UAS-myr::mRFP reveals that FRAP itself does not visibly affect visible filopodia structure (arrowhead). Pre, Prediffusion; Post, postdiffusion. $\boldsymbol{B}$, Individual data points from seven animals of each genotype were fit to $F(t)=1-f_{\mathrm{s}}-f_{\mathrm{f}} \mathrm{e}^{-t / \lambda}$, an equation used previously to document FRAP for actin::GFP in dendritic spines of transfected hippocampal neurons (Star et al., 2002). Actin::GFP turnover in UAS-CaMKII T287D ( $t_{1 / 2}$ $=132 \mathrm{~s} ; p<2 \times 10^{-6}$; black diamond) and UAS-Rac1 ( $t_{1 / 2} 102 \mathrm{~s} ; p<6 \times 10^{-6}$; red circle) animals dramatically increased compared with UAS-CaMKII T287A ( $t_{1 / 2}=347 \mathrm{~s}$; blue cross) or UAS-CaMKII animals (green triangles).

fewer short $(0-1.99 \mu \mathrm{m})$, filopodia than wild type (Fig. 4). It is possible that CaMKII T287A monomers integrate with endogenous CaMKII subunits in forming the holo-enzyme and reduce autophosphorylation by neighboring subunits to produce a dominantnegative effect. In fact, T287A substitutions in transgenic mice produce electrophysiological phenotypes slightly more severe than knock-outs of CaMKII- $\alpha$ themselves (Giese et al., 1998), suggesting a potential dominant-negative effect when mixed with other CaMKII isoforms in a multimeric enzyme. Expression of CaMKII protein variants in a characterized genetic-null background could test this hypothesis in the future.

How does protein localization regulate function? The activation state of CaMKII has been proposed to change the subcellular distribution of the protein by altering affinities for proteinbinding partners. In mammals, it is suggested that activated CaMKII displays an increased affinity for NMDA receptors (Shen and Meyer, 1999) and postsynaptic densities (PSDs) (Yoshimura and Yamauchi, 1997). We did not observe gross subcellular distribution changes between T-D and T-A protein variants in Drosophila da neurons (Fig. 3). There are several possible explanations for this observation. Immunostaining is not always quantitative, so it is possible that we could not detect small differences that did exist. Alternatively, the CaMKII T287A variant could multimerize with endogenous CaMKII subunits and become recruited to the same sites as autoactivated CaMKII. It is important to note that we have only characterized protein variant localization in fixed tissue. Expression of fluorescently tagged CaMKII variants in a protein null background could address whether different protein variants localize distinctly in real time in transgenic Drosophila.

\section{CaMKII and Rac1 function distinctly in} Drosophila dendrites

Given the known roles for Rho subfamily signaling on the actin cytoskeleton, we analyzed the roles of Racl signaling on dendritic structure in relationship to CaMKII (Figs. 3, 4, 7). In our studies, CaMKII modulates both morphological stability and cytoskeletal dynamics. In contrast, Racl signaling alters cytoskeletal dynamics without changing morphological stability. Racl activity greatly increases F-actin turnover as well, but the effects on structural plasticity are indistinguishable from CaMKII T287A or wild type (Figs. 4, 6). Importantly, whereas CaMKII increases the number of dendritic filopodia only on da class III neurons, Rac1 increases filopodia on all da neurons, even those that are normally devoid of filopodia (Figs. 3, 7). Although the morphology of filopodia on Rac1-expressing animals can be slightly unusual, unlike CaMKII T287D, the density on class III neurons is no greater than wild type (Fig. $3 \mathrm{~K}, \mathrm{O}$ ). The combination of phenotypic analysis, time lapse, and FRAP suggest that Racl and CaMKII distinctly regulate dendritic filopodia. Furthermore, $50 \%$ reduction of all rac genes (HakedaSuzuki et al., 2002; Ng et al., 2002) in heterozygous rac mutants did not suppress the CaMKII phenotype (data not shown). Expression of a dominant-negative Rac1 construct (N17) (Luo et al., 1994), which should block Rac-mediated GEF signaling, also failed to block the CaMKII T287D phenotype (data not shown). We also expressed both transgenes simultaneously, which does not result in increased densities of filopodia on non-class III neurons (data not shown) as might be predicted. This suggests either CaMKII is downstream of Racl or they modulate filopodia by distinct mechanisms.

An alternative hypothesis is that CaMKII activity strictly modulates the dynamics of dendritic filopodia extension and retraction, whereas Racl signaling preferentially modulates initial formation. There is evidence that the same pathways are used differently to regulate morphology in distinct subpopulations of neurons in mammals. Transgenic mice expressing dominantnegative p21-activated kinase (PAK) have abnormal dendritic spines in some cortical regions but not all regions (Hayashi et al., 2004). Furthermore, it has been demonstrated in Drosophila that many developmental defects can be rescued in rac triple-null mutants by a version lacking the effecter domain for PAK ( $\mathrm{Ng}$ et al., 2002), supporting different roles for downstream effectors in different cellular contexts. 
Implications for structural plasticity

In Drosophila, altering sensory neuron surface areas that function in thermosensation, mechanosensation, or regulating crawling behavior (Grueber et al., 2001; Ainsley et al., 2003; Liu et al., 2003; Tracey et al., 2003) would have profound consequences in transducing environmental cues. Changing the degree of coupling between morphology and the cytoskeleton could result in different elicited behaviors from the same environmental cue. In addition, preliminary observations suggest that larvae reared in different environments can result in filopodia with different morphologies (R. Andersen, P. Medina, Z. Blalock, and J. E. Brenman, unpublished observations). Changing environmental conditions could therefore regulate the dynamic nature or morphology of filopodia, thus controlling the animal's behavioral response.

What are the benefits of independently regulating morphological stability and cytoskeletal stability? In other systems, mechanisms to distinctly regulate cytoskeletal dynamics and morphological plasticity could be important during synaptogenesis, modification of synaptic strength, or neurite outgrowth. Calcium activation of multiple pathways, including CaMKII, could help sculpt morphological shape during periods of specialized neural activity. Evidence suggests synaptic strengthening is associated with morphological changes of dendritic spines into mushroom-shaped or perforated spine structures (Harris et al., 1992). One could imagine simultaneous activation and deactivation of numerous signaling pathways could sculpt a filopodium into a spine or vice versa. In fact, spines and filopodia have been directly observed to interconvert (Parnass et al., 2000; Roelandse et al., 2003). Spine motility and filopodia motility (Korkotian and Segal, 2001; Yuste and Bonhoeffer, 2001) could be modulated by differential signaling in pathways, including CaMKII and Rac, to adjust the likelihood of contacting other cells during development. Delivery and internalization rates of postsynaptic scaffolding/stabilizing proteins and receptors could be tied to cytoskeletal turnover and thus alter the ability to process neural information or transduce molecular signals. Another possibility is that receptor function could depend on physical anchoring to the actin cytoskeleton, which would serve as an indirect means of regulating receptor signaling without necessarily altering protein amount. Comparisons of cytoskeletal turnover and structural stability of dendritic compartments using FRAP, fluorescence resonance energy transfer, two-photon microscopy, and other in vivo techniques may allow direct comparisons of specific

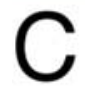

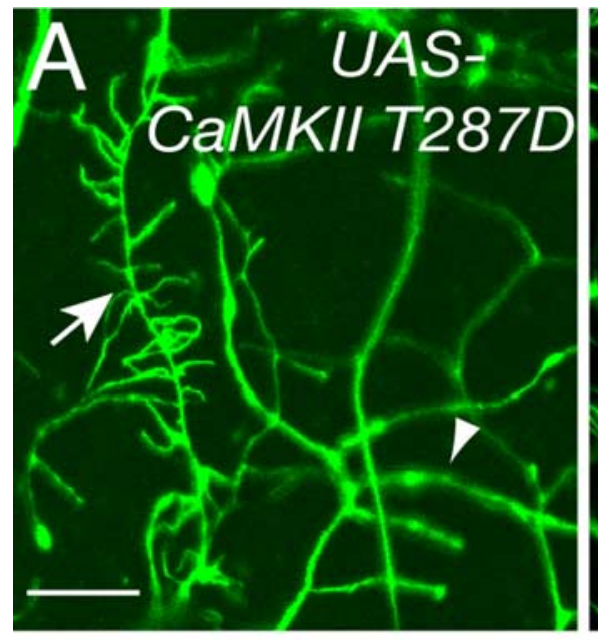
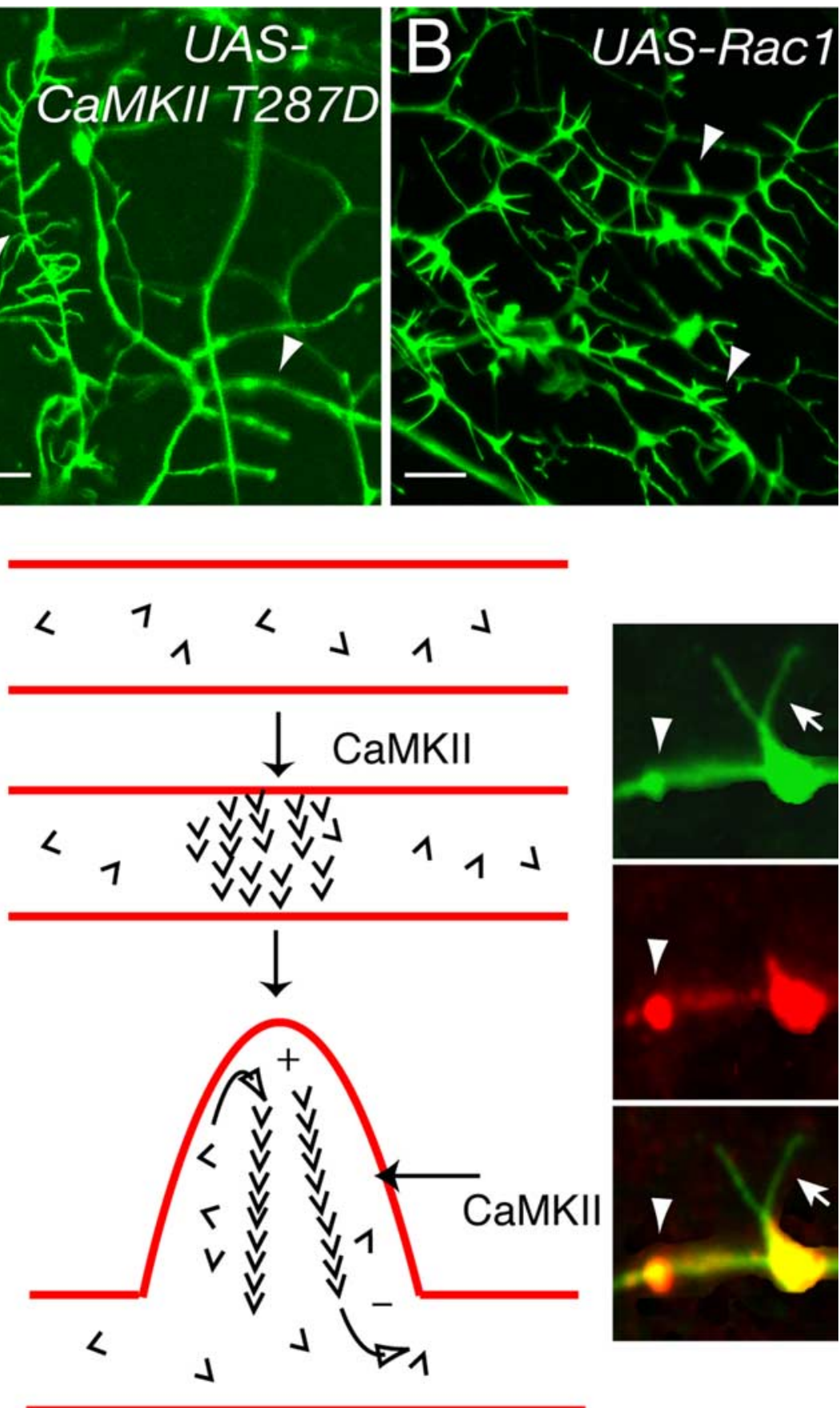

Figure 7. Rac1 and CaMKII modulate dendritic filopodia by distinct mechanisms. $A, B$, Analyzing dendrites of class I neuron $\operatorname{ddaE}(\boldsymbol{A}$, arrowhead) and class III neuron $\operatorname{ddaF}(\boldsymbol{A}$, arrow) demonstrates that CaMKII only affects neurons that normally contain filopodia $(\boldsymbol{A}$, arrow), leaving neighboring dendrites from nonfilopodia-containing neurons ( $\boldsymbol{A}$, arrowhead) unaffected. In contrast, Rac1 activity $(\boldsymbol{B})$ causes de novo dendritic filopodia formation on all dendritic arborization neurons regardless of class types ( $\boldsymbol{B}$, arrowheads). Scale bars, $5 \mu \mathrm{m}$. $\boldsymbol{C}$, Schematic model of dendritic effects mediated by activated CaMKII on dendritic filopodia and actin turnover. A patch of dendritic membrane uniquely accumulates small amounts of filamentous actin. This patch can be the site of future filopodia extension (Fig. 1E). Free actin monomers (barbs) are continuously added to the filaments at the plus $(+)$ end and can become free monomer by dissociation at the minus ( - ) end. CaMKII could increase the exchange rate of monomers to result in increased rates of fluorescence recovery after photobleaching and also increase the formation of actinmembrane patches. Inset, Colocalization of CaMKII (red) and actin:GFP (green) in dendrites. CaMKII accumulates in dendrites specifically at sites of F-actin enrichment and dendritic filopodia formation.

molecules in mammalian and Drosophila dendrites in the future. Modulating different aspects of neural architecture, by regulating distinct pathways, could provide the neuron flexibility to respond to changing developmental or environmental cues. Although Rac has many documented roles for modulating cellular morphol- 
ogy, less is known about Rac (and Rho GTPases) genetically in LTP. In contrast, CaMKII has many documented genetic roles for LTP but few known signaling pathways to regulate dendritic structure and the cytoskeleton. Genetically amenable systems assayed with in vivo approaches, as we demonstrate, should allow for such analysis.

\section{References}

Ainsley JA, Pettus JM, Bosenko D, Gerstein CE, Zinkevich N, Anderson MG, Adams CM, Welsh MJ, Johnson WA (2003) Enhanced locomotion caused by loss of the Drosophila DEG/ENaC protein Pickpocket1. Curr Biol 13:1557-1563.

Baas PW, Deitch JS, Black MM, Banker GA (1988) Polarity orientation of microtubules in hippocampal neurons: uniformity in the axon and nonuniformity in the dendrite. Proc Natl Acad Sci USA 85:8335-8339.

Bodmer R, Jan YN (1987) Morphological differentiation of the embryonic peripheral neurons in Drosophila. Rouxs Arch Dev Biol 196:69-77.

Brand AH (1995) GFP in Drosophila. Trends Genet 11:324-325.

Brand AH, Perrimon N (1993) Targeted gene expression as a means of altering cell fates and generating dominant phenotypes. Development 118:401-415.

Burton PR (1988) Dendrites of mitral cell neurons contain microtubules of opposite polarity. Brain Res 473:107-115.

Chen C, Rainnie DG, Greene RW, Tonegawa S (1994) Abnormal fear response and aggressive behavior in mutant mice deficient for alphacalcium-calmodulin kinase II. Science 266:291-294.

Clark IE, Jan LY, Jan YN (1997) Reciprocal localization of Nod and kinesin fusion proteins indicates microtubule polarity in the Drosophila oocyte, epithelium, neuron and muscle. Development 124:461-470.

Cline HT (2001) Dendritic arbor development and synaptogenesis. Curr Opin Neurobiol 11:118-126.

Dethier VG (1963) The physiology of insect senses. London: Wiley.

Dutta D, Bloor JW, Ruiz-Gomez M, VijayRaghavan K, Kiehart DP (2002) Real-time imaging of morphogenetic movements in Drosophila using Gal4-UAS-driven expression of GFP fused to the actin-binding domain of moesin. Genesis 34:146-151.

Emoto K, He Y, Ye B, Grueber WB, Adler PN, Jan LY, Jan YN (2004) Control of dendritic branching and tiling by the Tricornered-kinase/Furry signaling pathway in Drosophila sensory neurons. Cell 119:245-256.

Engert F, Bonhoeffer T (1999) Dendritic spine changes associated with hippocampal long-term synaptic plasticity. Nature 399:66-70.

Erondu NE, Kennedy MB (1985) Regional distribution of type II $\mathrm{Ca}^{2+}$ / calmodulin-dependent protein kinase in rat brain. J Neurosci 5:3270-3277.

Fifkova E, Delay RJ (1982) Cytoplasmic actin in neuronal processes as a possible mediator of synaptic plasticity. J Cell Biol 95:345-350.

Fink CC, Bayer KU, Myers JW, Ferrell Jr JE, Schulman H, Meyer T (2003) Selective regulation of neurite extension and synapse formation by the beta but not the alpha isoform of CaMKII. Neuron 39:283-297.

Fong YL, Taylor WL, Means AR, Soderling TR (1989) Studies of the regulatory mechanism of $\mathrm{Ca}^{2+} /$ calmodulin-dependent protein kinase II. Mutation of threonine 286 to alanine and aspartate. J Biol Chem 264:16759-16763.

Gao FB, Brenman JE, Jan LY, Jan YN (1999) Genes regulating dendritic outgrowth, branching, and routing in Drosophila. Genes Dev 13:2549-2561.

Giese KP, Fedorov NB, Filipkowski RK, Silva AJ (1998) Autophosphorylation at Thr286 of the alpha calcium-calmodulin kinase II in LTP and learning. Science 279:870-873.

Glazewski S, Chen CM, Silva A, Fox K (1996) Requirement for alphaCaMKII in experience-dependent plasticity of the barrel cortex. Science 272:421-423.

Grieder NC, de Cuevas M, Spradling AC (2000) The fusome organizes the microtubule network during oocyte differentiation in Drosophila. Development 127:4253-4264.

Grueber WB, Graubard K, Truman JW (2001) Tiling of the body wall by multidendritic sensory neurons in Manduca sexta. J Comp Neurol 440:271-283.

Grueber WB, Jan LY, Jan YN (2003) Different levels of the homeodomain protein cut regulate distinct dendrite branching patterns of Drosophila multidendritic neurons. Cell 112:805-818.

Hakeda-Suzuki S, Ng J, Tzu J, Dietzl G, Sun Y, Harms M, Nardine T, Luo L,
Dickson BJ (2002) Rac function and regulation during Drosophila development. Nature 416:438-442.

Hall A (1998) Rho GTPases and the actin cytoskeleton. Science 279:509-514.

Harris KM, Kater SB (1994) Dendritic spines: cellular specializations imparting both stability and flexibility to synaptic function. Annu Rev Neurosci 17:341-371.

Harris KM, Jensen FE, Tsao B (1992) Three-dimensional structure of dendritic spines and synapses in rat hippocampus (CA1) at postnatal day 15 and adult ages: implications for the maturation of synaptic physiology and long-term potentiation. J Neurosci 12:2685-2705.

Hayashi ML, Choi SY, Rao BS, Jung HY, Lee HK, Zhang D, Chattarji S, Kirkwood A, Tonegawa S (2004) Altered cortical synaptic morphology and impaired memory consolidation in forebrain-specific dominantnegative PAK transgenic mice. Neuron 42:773-787.

Hummel T, Krukkert K, Roos J, Davis G, Klambt C (2000) Drosophila Fut$\mathrm{sch} / 22 \mathrm{C} 10$ is a MAP1B-like protein required for dendritic and axonal development. Neuron 26:357-370.

Jan YN, Jan LY (2003) The control of dendrite development. Neuron 40:229-242.

Jin P, Griffith LC, Murphey RK (1998) Presynaptic calcium/calmodulindependent protein kinase II regulates habituation of a simple reflex in adult Drosophila. J Neurosci 18:8955-8964.

Jourdain P, Fukunaga K, Muller D (2003) Calcium/calmodulin-dependent protein kinase II contributes to activity-dependent filopodia growth and spine formation. J Neurosci 23:10645-10649.

Korkotian E, Segal M (2001) Spike-associated fast contraction of dendritic spines in cultured hippocampal neurons. Neuron 30:751-758.

Lee A, Li W, Xu K, Bogert BA, Su K, Gao FB (2003) Control of dendritic development by the Drosophila fragile X-related gene involves the small GTPase Rac1. Development 130:5543-5552.

Lee T, Winter C, Marticke SS, Lee A, Luo L (2000) Essential roles of Drosophila RhoA in the regulation of neuroblast proliferation and dendritic but not axonal morphogenesis. Neuron 25:307-316.

Lisman J, Schulman H, Cline H (2002) The molecular basis of CaMKII function in synaptic and behavioural memory. Nat Rev Neurosci 3:175-190.

Liu L, Yermolaieva O, Johnson WA, Abboud FM, Welsh MJ (2003) Identification and function of thermosensory neurons in Drosophila larvae. Nat Neurosci 6:267-273.

Luo L, Liao YJ, Jan LY, Jan YN (1994) Distinct morphogenetic functions of similar small GTPases: Drosophila Drac1 is involved in axonal outgrowth and myoblast fusion. Genes Dev 8:1787-1802.

Luo L, Hensch TK, Ackerman L, Barbel S, Jan LY, Jan YN (1996) Differential effects of the Rac GTPase on Purkinje cell axons and dendritic trunks and spines. Nature 379:837-840.

Maletic-Savatic M, Malinow R, Svoboda K (1999) Rapid dendritic morphogenesis in CAl hippocampal dendrites induced by synaptic activity. Science 283:1923-1927.

Matus A, Ackermann M, Pehling G, Byers HR, Fujiwara K (1982) High actin concentrations in brain dendritic spines and postsynaptic densities. Proc Natl Acad Sci USA 79:7590-7594.

Micheva KD, Vallee A, Beaulieu C, Herman IM, Leclerc N (1998) Beta-actin is confined to structures having high capacity of remodelling in developing and adult rat cerebellum. Eur J Neurosci 10:3785-3798.

Ng J, Nardine T, Harms M, Tzu J, Goldstein A, Sun Y, Dietzl G, Dickson BJ, Luo L (2002) Rac GTPases control axon growth, guidance and branching. Nature 416:442-447.

Niell CM, Meyer MP, Smith SJ (2004) In vivo imaging of synapse formation on a growing dendritic arbor. Nat Neurosci 7:254-260.

Parnass Z, Tashiro A, Yuste R (2000) Analysis of spine morphological plasticity in developing hippocampal pyramidal neurons. Hippocampus 10:561-568.

Pettit DL, Perlman S, Malinow R (1994) Potentiated transmission and prevention of further LTP by increased CaMKII activity in postsynaptic hippocampal slice neurons. Science 266:1881-1885.

Roelandse M, Welman A, Wagner U, Hagmann J, Matus A (2003) Focal motility determines the geometry of dendritic spines. Neuroscience 121:39-49.

Rubin GM, Spradling AC (1982) Genetic transformation of Drosophila with transposable element vectors. Science 218:348-353. 
Sabatini BL, Oertner TG, Svoboda K (2002) The life cycle of $\mathrm{Ca}^{2+}$ ions in dendritic spines. Neuron 33:439-452.

Scott EK, Luo L (2001) How do dendrites take their shape? Nat Neurosci 4:359-365.

Scott EK, Reuter JE, Luo L (2003) Small GTPase Cdc42 is required for multiple aspects of dendritic morphogenesis. J Neurosci 23:3118-3123.

Shen K, Meyer T (1999) Dynamic control of CaMKII translocation and localization in hippocampal neurons by NMDA receptor stimulation. Science 284:162-166.

Silva AJ, Stevens CF, Tonegawa S, Wang Y (1992a) Deficient hippocampal long-term potentiation in alpha-calcium-calmodulin kinase II mutant mice. Science 257:201-206.

Silva AJ, Wang Y, Paylor R, Wehner JM, Stevens CF, Tonegawa S (1992b) Alpha calcium/calmodulin kinase II mutant mice: deficient long-term potentiation and impaired spatial learning. Cold Spring Harb Symp Quant Biol 57:527-539.

Star EN, Kwiatkowski DJ, Murthy VN (2002) Rapid turnover of actin in dendritic spines and its regulation by activity. Nat Neurosci 5:239-246.

Takamatsu Y, Kishimoto Y, Ohsako S (2003) Immunohistochemical study of $\mathrm{Ca}^{2+} /$ calmodulin-dependent protein kinase II in the Drosophila brain using a specific monoclonal antibody. Brain Res 974:99-116.

Toni N, Buchs PA, Nikonenko I, Bron CR, Muller D (1999) LTP promotes formation of multiple spine synapses between a single axon terminal and a dendrite. Nature 402:421-425.

Tracey WD, Wilson RI, Laurent G, Benzer S (2003) Painless, a Drosophila gene essential for nociception. Cell 113:261-273.

Trachtenberg JT, Chen BE, Knott GW, Feng G, Sanes JR, Welker E, Svoboda $\mathrm{K}$ (2002) Long-term in vivo imaging of experience-dependent synaptic plasticity in adult cortex. Nature 420:788-794.
Verkhusha VV, Tsukita S, Oda H (1999) Actin dynamics in lamellipodia of migrating border cells in the Drosophila ovary revealed by a GFP-actin fusion protein. FEBS Lett 445:395-401.

Waldmann R, Hanson PI, Schulman H (1990) Multifunctional $\mathrm{Ca}^{2+}$ calmodulin-dependent protein kinase made $\mathrm{Ca} 2+$ independent for functional studies. Biochemistry 29:1679-1684.

Wang Z, Palmer G, Griffith LC (1998) Regulation of Drosophila $\mathrm{Ca}^{2+}$ / calmodulin-dependent protein kinase II by autophosphorylation analyzed by site-directed mutagenesis. J Neurochem 71:378-387.

Winter CG, Wang B, Ballew A, Royou A, Karess R, Axelrod JD, Luo L (2001) Drosophila Rho-associated kinase (Drok) links Frizzled-mediated planar cell polarity signaling to the actin cytoskeleton. Cell 105:81-91.

Wu GY, Cline HT (1998) Stabilization of dendritic arbor structure in vivo by CaMKII. Science 279:222-226.

Yoshimura Y, Yamauchi T (1997) Phosphorylation-dependent reversible association of $\mathrm{Ca}^{2+} /$ calmodulin-dependent protein kinase II with the postsynaptic densities. J Biol Chem 272:26354-26359.

Yu W, Cook C, Sauter C, Kuriyama R, Kaplan PL, Baas PW (2000) Depletion of a microtubule-associated motor protein induces the loss of dendritic identity. J Neurosci 20:5782-5791.

Yuste R, Bonhoeffer T (2001) Morphological changes in dendritic spines associated with long-term synaptic plasticity. Annu Rev Neurosci 24:1071-1089.

Zito K, Knott G, Shepherd GM, Shenolikar S, Svoboda K (2004) Induction of spine growth and synapse formation by regulation of the spine actin cytoskeleton. Neuron 44:321-334.

Zou DJ, Cline HT (1999) Postsynaptic calcium/calmodulin-dependent protein kinase II is required to limit elaboration of presynaptic and postsynaptic neuronal arbors. J Neurosci 19:8909-8918. 